\title{
Phylogeny and Histopathology of Streptococcus iniae
}

\section{from Indonesia}

\author{
Titis Candra Dewi ${ }^{1}$, Kurniasih $^{2}$, Surya Amanu ${ }^{3}$ and Rini Widayati ${ }^{4}$ \\ 1. Fish Quarantine and Inspection Agency, Semarang 50129, Indonesia \\ 2. Department of Pathology, Faculty of Veterinary Medicine, Gadjah Mada University, Yogyakarta 560862, Indonesia \\ 3. Department of Microbiology, Faculty of Veterinary Medicine, Gadjah Mada University, Yogyakarta 560862, Indonesia \\ 4. Department of Biochemistry, Faculty of Veterinary Medicine, Gadjah Mada University, Yogyakarta 560862, Indonesia
}

\begin{abstract}
Streptococcus iniae has been detected in several regions in Indonesia, such as Bali, Jambi and Papua. Outbreak of meningoencephalitis and septicemia was still often found in Papua. The aim of study was to compare phenotype, genotype and histopathology of S. iniae from Papua. Bacteria was identified using morphological and biochemical tests. It was also extracted, amplified and sequenced to see genotypic characters. The primer used were 5'-AGAGTTTGATCCTGGCT-3' (24F) and 5'-AAGGGAGGTGATCCAGCCGCA-3' (1540R) in 16S rRNA region. Result of sequencing was analyzed by the neighbor joining and maximum parsimony methods. The identification result of isolate from Papua was $S$. iniae. The amplification result was a sharp band $-1,500 \mathrm{bp}$ band in $16 \mathrm{~S}$ rRNA region. The phylogenetic tree showed that isolate from Papua was closely related to $S$. iniae strains CMS005 from Guangxi China. Bacterial clumps of $S$. iniae was firstly found in the blood vessel of liver at the 3rd day after infection and then caused the inflammation to spleen, heart, brain, kidney and gut at the 7 th day post infection.
\end{abstract}

Key words: Phenotype, genotype, phylogeny.

\section{Introduction}

Streptococcus iniae is not only one of the major causative agent of streptococcosis in aquaculture industry but also an important zoonotic bacterial disease causing morbidity and mortality in humans. The emergence of disease has occurred in a range of aquatic animals [1]. The disease has been reported in almost all continents and has caused significant losses in several commercial fish species [2]. The estimated annual impact of disease outbreaks by $S$. iniae in aquaculture sector of some countries was reported to be 100 million USD [3].

S. iniae was first isolated from multifocal subcutaneous abscesses in captive Amazon freshwater dolphins-Iniae geoffrensis [4]. In fishes, S. iniae infections were characterized by meningoencephalitis and septicemia that generally

Corresponding author: Kurniasih, professor, research field: fish pathology. E-mail: kurniasih_1951@yahoo.co.id. induce high morbidity and mortality rates [1]. Clinical signs of $S$. iniae infection in fish include loss of orientation, lethargy, ulcers, exophthalmia and culminate in a fatal meningoencephalitis [1]. S. iniae can cause serious zoonotic infections in humans who are injured while handling death fish [5]. To date, this disease has been found in Indonesia farming of tilapia (Oreochromis niloticus) in Lubuk Linggau, Southern Sumatra during 2002-2003 [6] as well as in Lake Maninjau in 2010 [7]. S. iniae has been detected in several regions in Indonesia, such as Bali, Jambi and Papua [8]. Outbreak of meningoencephalitis and septicemia is still often found in Papua. Thus, no detailed description of $S$. iniae infection was reported until now. The aim of study was to describe the phenotypic and genotypic characteristic of $S$. iniae on the basis of $16 \mathrm{~S}$ rDNA sequences and histopathological changes. 


\section{Materials and Methods}

\subsection{Bacterial Isolation and Identification}

Streak of brain and kidney of tilapia with clinical signs exophthalmia and abdominal distention, was isolated onto $5 \%$ sheep blood agar and incubated at $28^{\circ} \mathrm{C}$ for $72 \mathrm{~h}$. The colonies obtained were tested by hemolysis, gram staining, catalase and oxidase production, then continue to identify the isolate using several bacterial cultures, such as TSA $6.5 \% \mathrm{NaCl}$, Bile esculin, Simon citrate, Methyl red and Voges-Proskaeuer (MR/VP), lysin decarboxylase, urea, motility indole ornithine (MIO), McConkey, glucose, lactose, maltose, sucrose, xylose, arabinose and mannitol [1]. All identification was compared with isolated of S. iniae (ATCC 29177). The classification method used parameters as described in bacterial fish pathogen [2].

\subsection{Molecular Studies}

Isolates of S. iniae from Papua and S. iniae (ATCC 29177) were extracted using DNA Extraction Kit (Qiagen). The primers used were 5'-AGAGTTTGATCCTGGCT-3' (24F) and 5'-AAGGGAGGTGATCCAGCCGCA-3' (1540R) in 16S rRNA region. A reaction matrix of $25 \mu \mathrm{L}$ contained 12.5 $\mu \mathrm{L}$ master mix (Roche, Switzerland), $10 \times 10^{-12}$ mol concentration of each primer, $6.5 \mu \mathrm{L}$ steril $\mathrm{ddH}_{2} \mathrm{O}$ and $4 \mu \mathrm{L}$ DNA template. Typical cycling parameters were: 5 min primary denaturation at $95^{\circ} \mathrm{C}$, $30 \mathrm{~s}$ denaturation at $92{ }^{\circ} \mathrm{C}, 90 \mathrm{~s}$ annealing at $52{ }^{\circ} \mathrm{C}, 60$ $\mathrm{s}$ extension at $72{ }^{\circ} \mathrm{C}$ for 30 cycles and then 5 min final extension step at $72{ }^{\circ} \mathrm{C}$. The polymerase chain reaction $(\mathrm{PCR})$ products were then electrophoresed in $1 \%$ gel agarose. Purification and sequencing of PCR product were done in first base Singapore.

\subsection{Alignment and Phylogeny Analysis}

Sequence results were compared to $16 \mathrm{~S}$ rRNA sequences that are available in the GenBank/EMBL/DDBJ databases using the Basic
Local Alignment Search Tool (BLAST) algorithm [9]. Multiple sequence alignment of representative sequences was carried out by using the CLUSTAL $\mathrm{X}$ program (version 1.8) [10]. Other sequences of the following streptococcal species are available in the National Center for Biotechnology Information (NCBI) database: $S$. iniae strains WZMH110819 from Wenzhou, China; S. iniae strains SK10-S from Sentani, Papua; S. iniae strains SF2 from Iran; S. iniae strains SF1 from China; $S$. iniae strains SCCF5L from China; S. iniae from Japan; $S$. iniae from Israel; $S$. iniae strains ISET0901 from Israel; $S$. iniae strains DGX01 from China; S. iniae strains CMS005 from Guangxi, China; S. iniae strains I1 from Japan; and Streptococcus hongkongensis strains HKU30, Streptococcus thermophilus, Staphylococcus aureus subsp. aureus strains $\mathrm{CN} 1$ and isolates of S. iniae from Papua. The genetic distance matrix was obtained using Kimura's two parameter model [11] and an evolutionary tree was created using the neighbor joining and maximum parsimony methods [12] with MEGA 6 [13]. Bootstrap values from 1,000 replicates are displayed as percentages.

\subsection{Pathogenicity Test}

Thirty of tilapias were injected intraperitoneally with $0.1 \mathrm{~mL} \times 10^{6} \mathrm{CFU} /$ fish. Five fish were killed at 3, $5,7,9$ and 11 days post infection to examine the histopathological changes. Tissue samples were fixed in $10 \%$ solution of neutral buffered formalin and then stained with haematoxylin and eosin (H\&E).

\section{Results and Discussion}

\subsection{Phenotypic Result}

Affected fish were loss of equilibrium, erratic swimming which is either spiralling or spinning just below the surface of water, darkening, exopthalmus, petechiae on the operculum and fins. At autopsy, ascites, hemorhage of the internal organs and enlarged spleens were found.

In this study, the isolate was $\beta$-haemolytic, gram 
positive, catalase negative and oxidase negative. The isolate showed negative reaction in voges proskauer and positive reaction in esculin. The isolate grew on MacConkey agar at $37{ }^{\circ} \mathrm{C}$, but did not grow on $6.5 \%$ $\mathrm{NaCl}$. The isolate had a negative reaction for lactose, inositol, arabinose, dulcitol, sorbitol, raffinose and inulin. Positive reactions were observed in glucose, maltose, mannitol and mannose. This result was similar to identification made in Ref. [2] and to strain ATCC 29177.

The identification results of isolate were biochemically and physiologically similar to $S$. iniae. The taxonomic position of many aquatic isolates is still controversial [2]. Standard bacteriological methods were not adequate for identification of the fish pathogen S. iniae. Moreover, the S. iniae could not be identified by most commercial bacterial identification [4], because it was not listed in the databases of the most commonly used rapid or automated identification systems, including the Rapid Strep Strip, Vitex systems, API 20E Strep, Rapid Strep 32, API CH 50 or the ATB expression systems [14-16].

\subsection{Phylogeny of S. iniae from Papua}

Phylogenetic tree of S. iniae using neighbour-joining
(Fig. 1) showed that it was clustered to other S. iniae from several countries, except for isolate of $S$. hongkongensis HKU30 and S. thermophilus.

Phylogenetic tree of $S$. iniae using maximum parsimony (Fig. 2) showed that S. iniae from Papua was grouped in the same clade with S. iniae CMS005 Guangxi China and S. iniae DGX01 China.

Homology search of the 16S rRNA sequence $(1,500$ bp) of strain $S$. iniae from Sentani (GenBank/EMBL/DDBJ databases) showed 84\% similarity with strain $S$. iniae from Papua using maximum parsimony and $100 \%$ similarity using neighbour-joining.

Then, S. iniae from Papua was closest to S. iniae CMS005 Guangxi China, despite the low validity (19\% similarity using maximum parsimony analysis).

Specific PCR primer sequences have been developed as a useful alternative approach for the accurate and rapid identification of $S$. iniae, such as the 16S rRNA gene [17]. Genotypic identification was useful in the identification of carrier fish [18]. In this study, $84 \%$ similarity using maximum parsimony was shown in the sequences of the 16S rRNA gene between the $S$. iniae isolate (Papua, Indonesia) and the S. iniae CMS005 (Guangxi China).

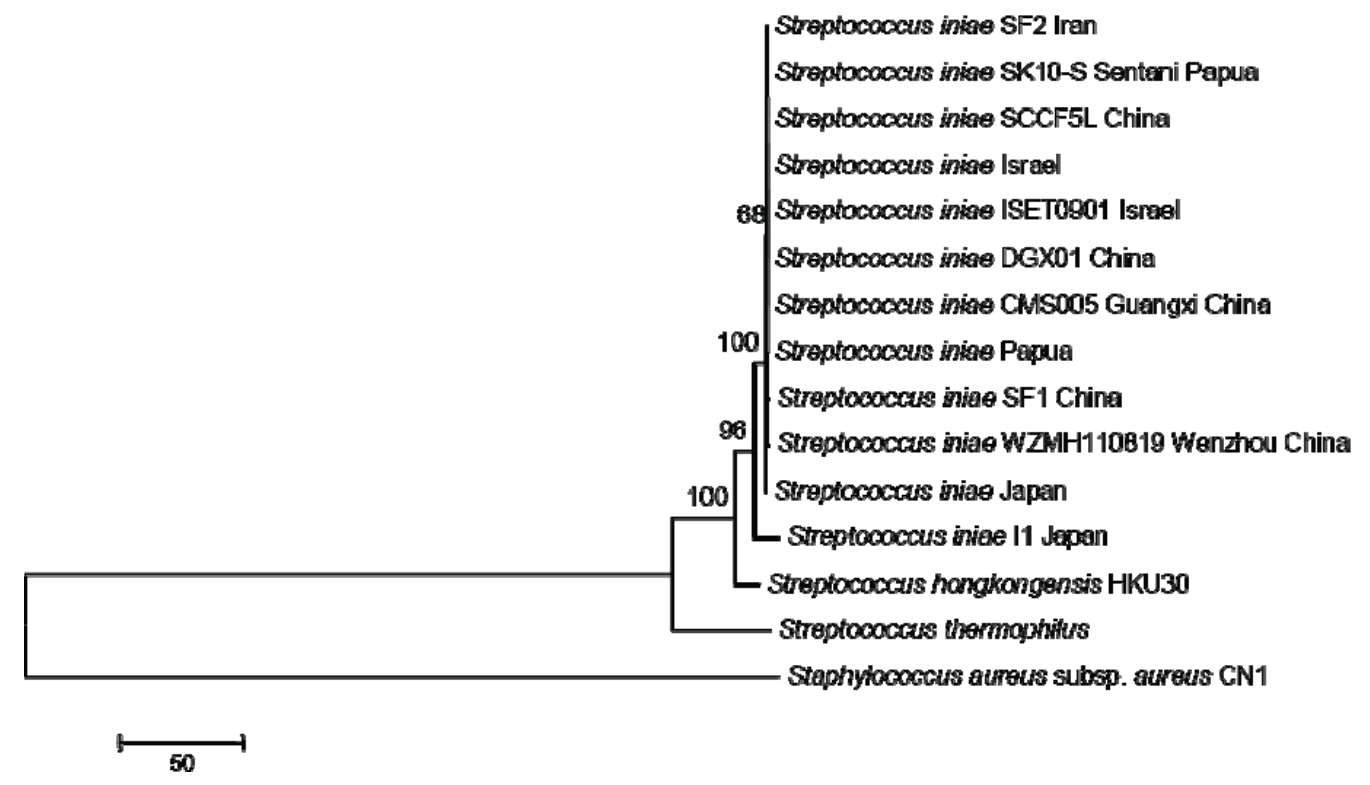

Fig. 1 Phylogenetic tree of $S$. iniae from Papua using neighbour-joining analysis. 


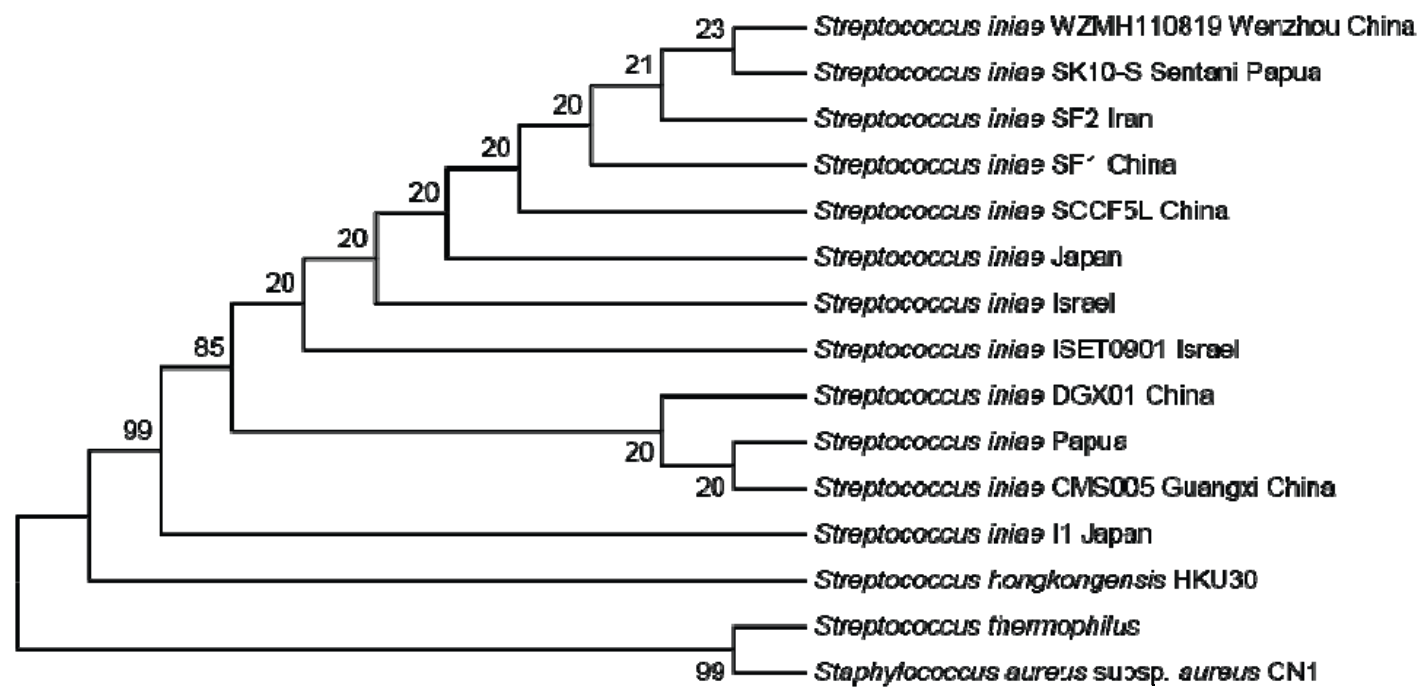

Fig. 2 Phylogenetic tree of $S$. iniae from Papua using maximum parsimony analysis.

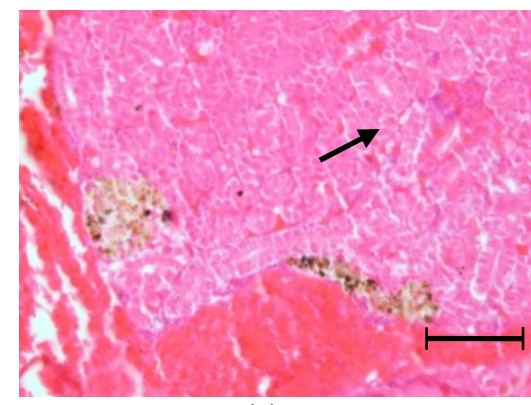

(a)

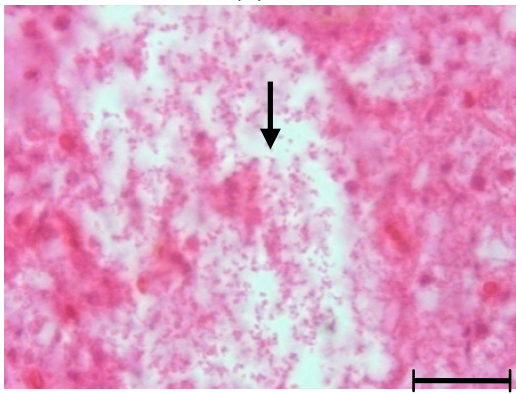

(c)

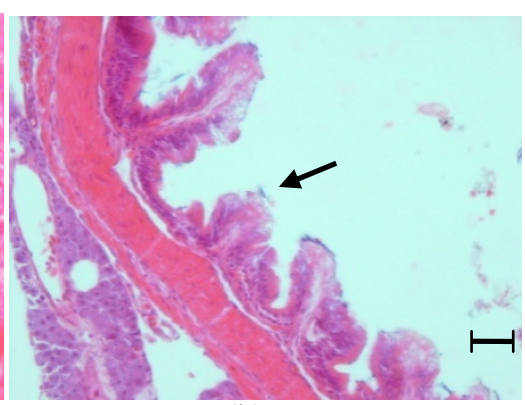

(b)

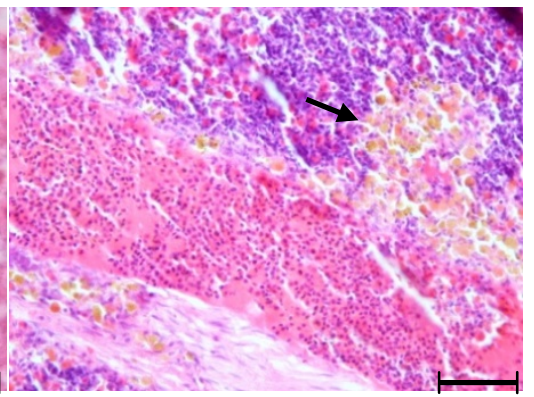

(d)

Fig. 3 Increasing of renal melanomacrophage (a), necrosis (arrow) of epithelial gut (b), bacterial clumps (arrow) in the blood vessel of liver (c), congestion and inflammation in the spleen (d).

Scale bar: $20 \mu \mathrm{m}$.

\subsection{Clinical Signs}

Fish began to show clinical signs and morbidity at three days post infection with S. iniae. Fish showed signs of disorientation, anorexic, erratic swimming and listless circling at the water surface. Some of the fish had exophthalmia. Macroscopics lesions at three days post infection were darkness of skin, external hemorrhages especially around the base of the pectoral fins and in the opercula. These observations were in accordance with the report in other studies; darkening of the skin and lethargy were the first signs observed in infected fish. Moribund and dead fish presented external hemorrhages especially around the base of the pectoral fins and over the internal organs [19]. Clinical signs of streptococcal infections varied between species. Darkening of the skin pigment has 


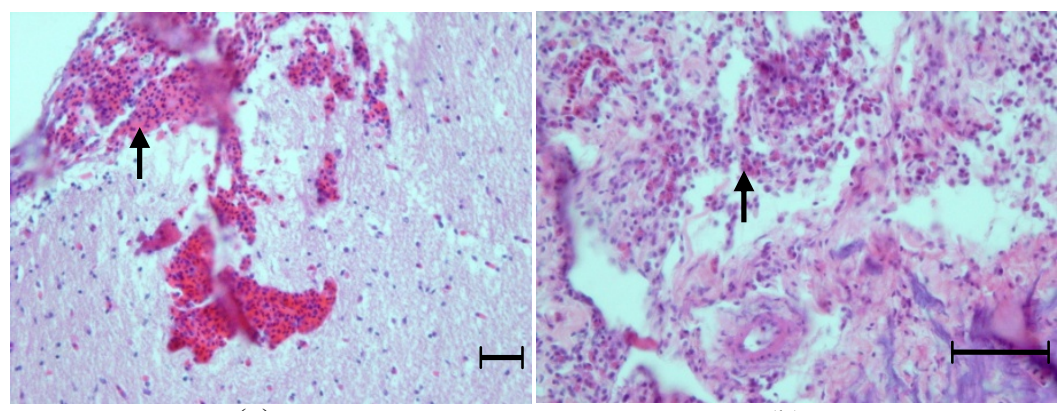

(a)

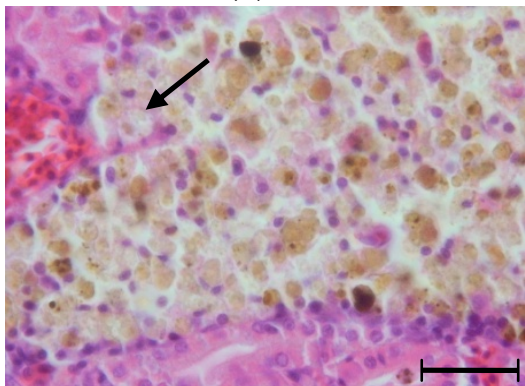

(c) (b)

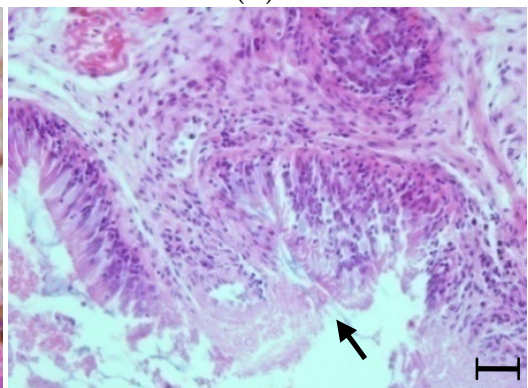

(d)

Fig. 4 Brain membrane was haemorhage (a), myocarditis (b), bacterial clumps within renal macrophage (c), erotion (arrow) and necrosis of mucosal gut (d).

Scale bar: $20 \mu \mathrm{m}$.

been reported in various fish species infected with Streptococcus sp. [20-22].

\subsection{Histopathological Changes}

Histopathological changes were found in several organs including spleen, heart, brain, liver, ren and gut. It was found the increasing of renal melanomacrophage, necrosis of epithelial gut, bacterial clumps in the blood vessel of liver and congestion and inflammation in the spleen at three days post infection (Fig. 3). It was more severe to find haemorhage of meningitis, myocarditis, bacterial clumps within renal macrophage, erotion and necrosis of mucosal gut at seven days post infection (Fig. 4).

$S$. iniae infection in fish is systemic infection of the liver, heart and brain. The histopathology examination found the infiltration of large numbers of increasing melanomacrophage in infected areas, such as the ren and spleen. Multifocal infiltration of macrophage cells in the kidney and spleen as the principal lesions, was also reported by other authors [23-25]. These findings can be correlated with the clinical findings of lethargy and loss of orientation [24]. Necrosis was similar to observation in tilapia infected with Streptococcus sp. [19]. Externally, Streptococcus sp. infection can cause lesions, congestions, hemorrhages, exophthalmos, corneal opacity, intra-ocular and periorbital hemorrhages. Due to the high capability of spleen and posterior kidney to trap bacteria, Streptococci can be commonly found in those organs in conjuction to tissue necrosis [26].

\section{Conclusions}

In this study, isolates from Papua were closely related to Chinese isolate (DGX01, Guangxi region). Besides, bacterial clumps of $S$. iniae was firstly found in the blood vessel of liver at the 3rd day after infection and caused the inflammation to spleen, heart, brain, kidney and gut.

\section{References}

[1] Agnew, W., and Barnes, A. C. 2007. "Streptococcus iniae: An Aquatic Pathogen of Global Veterinary Significance and a Challenging Candidate for Reliable Vaccination." Vet. Microbiol: 122 (1-2): 1-15.

[2] Austin, B., and Austin, D. A. 2007. Bacterial Fish Pathogen: Disease of Farmed and Wild Fish, 4th ed.. 
Chichester, UK: Pringer-Praxis Publishing, 16-8.

[3] Shoemaker, C. A., Klesius, P. H., and Evans, J. J. 2001. "Prevalence of Streptococcus iniae in Tilapia, Hybrid Striped Bass and Channel Catfish on Commercial Fish Farms in the United States." Am. J. Vet. Res. 62 (2): 174-7.

[4] Pier, G. B., and Madin, S. H. 1976. "Streptococcus iniae sp. Nov.: A Beta-Hemolytic Streptococcus Isolated from an Amazon Freshwater Dolphin-Inia geoffrensis." Int. J. Sys. Bacter. 26 (4): 545-53.

[5] Weinstein, M. R., Litt, M., Kertesz, D. A., Wyper, P., Rose, D., Coulter, M., McGerr, A., Facklam, R., Ostach, C., Willey, B. M., Borczyk, A., and Low, D. E. 1997. "Invasive Infections Due to a Fish PathogenStreptococcus iniae." The New England Journal of Medicine 337 (9): 589-94.

[6] Yuasa, K., Kitancharoen, N., Kataoka, Y., and Al-Murbaty, F. A. 1999. "Streptococcus iniae: The Causative Agent of Mass Mortality in Rabbit Fish Siganus canaliculatus in Bahrain.” J. Aqua. Anim. Health 11 (1): 87-93.

[7] Supriyadi, H., and Gardenia, L. 2010. "Streptococcosis on Tilapia (Oreochromis niloticus) Cultivation in Lake Maninjau." In Proceedings of the Aquaculture Technology Innovation Forum, 905-10.

[8] Fish Quarantine and Inspection Agency. 2013. The Monitoring Results of Pest and Fish Disease by Fish Quarantine and Inspection Agency in 2013. Fish Disease Monitoring Report of Marine and Fisheries Ministry, Jakarta.

[9] Altschul, S. F., Warren, G., Webb, M., Eugene, W. M., and David, J. L. 1990. "Basic Local Alignment Search Tool.” J. Mol. Biol. 215 (3): 403-10.

[10] Thompson, J. D., Gibson, T. J., Plewniak, F., Jeanmougin, F., and Higgins, D. G. 1997. "The CLUSTAL X Windows Interface: Flexible Strategies for Multiple Sequence Alignment Aided by Quality Analysis Tools." Nucleic Acids Res. 25 (24): 4876-82

[11] Kimura, H., and Kusuda, R. 1979. "Studies on the Pathogenesis of Streptococcal Infection in Cultured Yellow Tails Seriola spp.: Effect of the Cell Free Culture on Experimental Streptococcal Infection." Journal Fish Dis. 2 (6): 501-10.

[12] Saitou, N., and Nei, M. 1987. "The Neighbor-Joining Method: A New Method for Reconstructing Phylogenetic Trees.” Mol. Biol. Evol. 4 (4): 406-25.

[13] Tamura, K., Dudley, J., Nei, M., and Kumar, S. 2007. "MEGA 4: Molecular Evolutionary Genetics Analysis (MEGA) Software Version 4.0.” Mol. Biol. Evol. 24 (8): 1596-9.

[14] Dodson, S. V., Maurer, J. J., and Shotts, E. B. 1999. "Biochemical and Molecular Typing of Streptococcus iniae Isolated from Fish and Human Cases." Journal of Fish Diseases 22 (5): 331-6.

[15] Lau, S. K. P., Woo, P. C. Y., Tse, H., Leung, K. W., Wong, S. S. Y., and Yuen, K. Y. 2003. "Invasive Streptococcus iniae Infections outside North America." Journal of Clinical Microbiology 41 (3): 1004-9.

[16] Roach, J. C., Levett, P. N., and Lavoie, M. C. 2006. "Identification of Streptococcus iniae by Commercial Bacterial Identification Systems." Journal of Microbiological Methods 67 (1): 20-6.

[17] Berridge, B. R., Fuller, J. D., Azavedo, J., Low, D. E., Bercovier, H., and Frelier, F. 1998. "Development of a Specific Nested Oligonucleotide PCR Primer for S. iniae 16S-23S Ribosomal DNA Intergenic Spacer.” Journal of Clinical Microbiology 36: 2778-81.

[18] Zlotkin, A., Hershko, H., and Eldar, A. 1998. "Possible Transmission of Streptococcus iniae from Wild Fish to Cultured Marine Fish." Applied and Environmental Microbiology 64 (10): 4065-7.

[19] Mehdi, R., Sasan, Z., Arman, A., Seyed, F., and Firouz, F. 2012. "Experimental Evaluation of Pathogenicity of Streptococcus iniae in Silver Shark and Rainbow Shark." African Journal of Microbiology Research 6 (14): 3560-3.

[20] Bragg, R. R. 1988. "The Indirect Fluorescent Antibody Technique for the Rapid Identification of Streptococcosis of Rainbow Trout (Salmo gairdneri)." Onderstepoort J. Vet. Res. 55 (1): 59-61.

[21] Evans, J. J., Shoemaker, C. A., and Klesius, P. H. 2000. "Experimental Streptococcus iniae Infection of Hybrid Striped Bass (Morone chrysops $\times$ Morone saxatilis) and Tilapia (Oreochromis niloticus) by Nares Inoculation." Aquaculture 189 (3-4): 197-210.

[22] Shoemaker, C. A., Evans, J. J., and Klesius, P. H. 2000. "Density and Dose: Factors Affecting Mortality of Streptococcus iniae Infected Tilapia (Oreochromis niloticus)." Aquaculture 188 (3): 229-35.

[23] Bercovier, H., Ghittino, C., and Eldar, A. 1996. "Immunization with Bacterial Antigens: Infections with Streptococci and Related Organisms." Dev. Biol. Stand. 90: $153-60$.

[24] Eldar, A., and Ghittino, C. 1999. "Lactococcus garvieae and Streptococcus iniae Infection in Rainbow Trout Oncorhynchus mykiss: Similar, but Different Diseases." Dis. Aquat. Org. 36 (3): 227-31.

[25] Bromage, E. S., and Owens, L. 2002. "Infection of Barramundi Lates calcarifer with Streptococcus iniae: Effects of Different Routes of Exposure." Dis. Aquat. Org. 52 (3): 199-205.

[26] Miyazaki, T., Kubota, S. S., Kaige, N., and Miyashita, T. 1984. "A Histopathological Study of Streptococcal Disease in Tilapia." Fish Pathology 19 (3): 167-72. 